\title{
Impact of new physics on the EW vacuum stability in a curved spacetime background
}

\author{
E. Bentivegna, ${ }^{a, b}$ V. Branchina, ${ }^{a, b}$ F. Contino ${ }^{a, c}$ and D. Zappalà ${ }^{b}$ \\ ${ }^{a}$ Department of Physics and Astronomy, University of Catania, \\ Via Santa Sofia 64, Catania, 95123 Italy \\ ${ }^{b}$ INFN, Sezione di Catania, \\ Via Santa Sofia 64, Catania, 95123 Italy \\ ${ }^{c}$ Scuola Superiore di Catania, \\ Via Valdisavoia 9, Catania, 95123 Italy \\ E-mail: eloisa.bentivegna@ct.infn.it, branchina@ct.infn.it, \\ contino.filippo92@gmail.com, dario.zappala@ct.infn.it
}

ABSTRACT: It has been recently shown that, contrary to an intuitive decoupling argument, the presence of new physics at very large energy scales (say around the Planck scale) can have a strong impact on the electroweak vacuum lifetime. In particular, the vacuum could be totally destabilized. This study was performed in a flat spacetime background, and it is important to extend the analysis to curved spacetime since these are Planckian-physics effects. It is generally expected that under these extreme conditions gravity should totally quench the formation of true vacuum bubbles, thus washing out the destabilizing effect of new physics. In this work we extend the analysis to curved spacetime and show that, although gravity pushes toward stabilization, the destabilizing effect of new physics is still (by far) the dominating one. In order to get model independent results, high energy new physics is parametrized in two different independent ways: as higher order operators in the Higgs field, or introducing new particles with very large masses. The destabilizing effect is observed in both cases, hinting at a general mechanism that does not depend on the parametrization details for new physics, thus maintaining the results obtained from the analysis performed in flat spacetime.

Keywords: Beyond Standard Model, Higgs Physics, Nonperturbative Effects, Solitons Monopoles and Instantons

ArXiv EPrint: 1708.01138 


\section{Contents}

1 Introduction 1

2 Theoretical background 4

3 New physics: higher order operators $\quad 9$

4 New physics: fermions and bosons with large masses 13

$\begin{array}{llr}5 & \text { Summary, conclusions, and outlook } & 17\end{array}$

$\begin{array}{ll}\text { A Numerical computation of the bounce solution } & 18\end{array}$

\section{Introduction}

One of the most important goals of present theoretical and experimental particle physics is the search for New Physics (NP) beyond the Standard Model (BSM), even though direct experimental searches up to now have not revealed any sign of it. When looking for patterns towards BSM theories, the stability analysis [1-15] of the electroweak (EW) vacuum plays a crucial role. Earlier studies were mainly focused on establishing bounds for the Higgs boson mass, based either on the requirement that the Higgs effective potential $V(\phi)$ could not take values lower than the EW minimum $v$ (so that the latter is the stable vacuum of the theory) or on the possibility that our Universe sits on a metastable (false) vacuum state (i.e. $V(v)$ is not the absolute minimum of $V(\phi)$ ), with a lifetime larger than its own age $[2,11,16,17]$.

The discovery of the Higgs boson boosted new interest on the stability problem. Clearly the goal is no longer to derive bounds on its mass, but rather to perform more refined analyses that should allow to discriminate between absolute stability or metastability for the EW vacuum [18-21], to study the cosmological impact of the vacuum stability condition during and after inflation [22-32], and to test the impact that different NP scenarios can have on the vacuum stability condition [18,33-44]. This renewed interest also prompted a more careful treatment of issues as the gauge invariance of the vacuum decay rate and the contribution of zero modes to the quantum fluctuation determinant [45-50].

On the theoretical side, the stability analysis has its roots in a pioneering work of Bender and collaborators [51,52], where the tunneling for a quantum mechanical system with several degrees of freedom was studied with the help of saddle point techniques. This was later extended to quantum field theory by Coleman and Callan, who studied the decay of the false vacuum in a flat spacetime background [53, 54], and then by Coleman and De Luccia [55], who included gravity in their analysis. 
Physically the false vacuum decay is triggered by quantum fluctuations, that induce a finite probability for a bubble of true vacuum to materialize in a false vacuum sea. Both in flat and curved spacetime backgrounds, Coleman and collaborators considered a scalar theory where the potential $V(\phi)$ has a relative and an absolute minimum, at $\phi_{\text {false }}$ and $\phi_{\text {true }}$ respectively, such that the energy density difference $V\left(\phi_{\text {false }}\right)-V\left(\phi_{\text {true }}\right)$ is much smaller than the height of the "potential barrier" $V\left(\phi_{\text {top }}\right)-V\left(\phi_{\text {false }}\right)$, where $V\left(\phi_{\text {top }}\right)$ is the maximum of the potential between the two minima. Under these conditions the true vacuum bubble is separated from the false vacuum sea by a "thin wall", and this allows to treat the problem analytically, within the so called "thin wall" approximation.

Going back to the SM, it is known that due to the top loop corrections the Higgs potential $V(\phi)$ turns over for values of $\phi>v$, where $v \sim 246 \mathrm{GeV}$ is the location of the EW minimum, and for the present experimental values of $M_{H}$ and $M_{t}$, namely $M_{H} \sim$ $125.09 \mathrm{GeV}$ and $M_{t} \sim 173.34 \mathrm{GeV}[56,57]$, it develops a second minimum, much deeper than the EW one and at a much larger value of the field, $\phi_{\text {true }} \gg v=\phi_{\text {false }}$. The instability scale [1] is defined as the value $\phi_{\text {inst }}$ of the field such that $V\left(\phi_{\text {inst }}\right)=V(v)$ and $V(\phi)<V(v)$ for $\phi>\phi_{\text {inst. }}$. For the values of the Higgs and top masses reported above, it turns out that $\phi_{\text {inst }} \sim 10^{11} \mathrm{GeV}$. Clearly the conditions under which the thin wall approximation can be applied are not fulfilled in the SM case, so the results of [53, 54] and [55] cannot be directly applied.

The EW vacuum stability condition was first studied in a flat spacetime background, and the interesting possibility that the $\mathrm{SM}$ is valid all the way up to the Planck scale $M_{\mathrm{P}}$, meaning that NP shows up only at this scale, was investigated. In such a scenario, naturally prompted by the lack of direct observation of hints of new physics, the analysis was performed under the assumption [11] that the presence of NP at $M_{\mathrm{P}}$ could be neglected for the computation of the tunneling time $\tau$ from the false to the true vacuum of the SM, so that $\tau$ was calculated by considering SM interactions only [11, 12, 18, 33-37]. In particular, in [12] it was argued that the reason why NP at $M_{\mathrm{P}}$ (even if present) can be neglected in the calculation of the tunneling rate is due to the fact that the instability scale $\phi_{\text {inst }} \sim 10^{11} \mathrm{GeV}$ is sufficiently smaller than $M_{\mathrm{P}}$, i.e. a decoupling effect was expected.

As it was later realized, the assumption that NP lives only at the scale $M_{\mathrm{P}}\left(\gg \phi_{\text {inst }}\right.$ ) does not imply that the latter cannot affect the stability condition of the EW vacuum. ${ }^{1}$ On the contrary, the latter is very sensitive to unknown NP even if it lives at scales far away from $\phi_{\text {inst }}$, and the decay rate of the EW vacuum can be strongly modified by its presence [38-40, 43].

The reason why the decoupling theorem does not hold in this case is that tunneling is a non-perturbative phenomenon [43], while the former applies when calculating scattering amplitudes in perturbation theory at energies $E$ much lower than $M_{\text {cut }}$, the physical cut-off scale of the theory under investigation $\left(M_{\mathrm{P}}, M_{\mathrm{GUT}}, \ldots\right)$. In this case the contributions to scattering amplitudes from physics that lives at $M_{\text {cut }}$ is suppressed by factors of $E / M_{\text {cut }}$ to

\footnotetext{
${ }^{1}$ It is worth to note that the relevant scale for the problem under investigation is not $\phi_{\text {inst }}$ but rather the scale $\phi^{*}$ where the renormalization group improved quartic coupling $\lambda(\phi)$ is such that $d \lambda / d \phi=0$, as this is the scale where the probability of the bubble nucleation is maximized [58].
} 
the appropriate power, and this is how physics at the scale $M_{\text {cut }}$ is decoupled from physics at the scale $E$.

For our tunneling phenomenon however, the bulk of the contribution to $\tau$ comes from the exponential of the (Euclidean) action calculated at the saddle point of the path integral for the tunneling rate, the so called bounce solution to the (Euclidean) Euler-Lagrange equation [53,54], and for this tree level contribution no suppression factors of the kind $\left(E / M_{\mathrm{P}}\right)^{n}$ can ever appear. If the Higgs potential is modified by the presence of NP at $M_{\mathrm{P}}$, the new bounce is certainly different from the one obtained when these terms are neglected. The action calculated for this new bounce solution is also modified and (once exponentiated) it can give rise to a value of $\tau$ enormously different from the one obtained when the NP terms are neglected.

The inclusion of gravity in the vacuum stability analysis was pioneered in [55], where the case of the thin wall regime was studied. For the transition from a false Minkowski vacuum to a true Anti-de Sitter (AdS) vacuum, it was shown that, when the size of the Schwarzschild radius of the true vacuum bubble is much smaller than its size, i.e. when gravitational effects are weak, the probability of materialization of such a bubble is close to the flat spacetime result, while when the Schwarzschild radius becomes comparable to the bubble size, i.e. in a strong gravitational regime, the presence of gravity stabilizes the false vacuum, preventing the materialization of a true vacuum bubble. In other words, gravity tends to stabilize the false vacuum, and in a strong gravity regime the materialization of bubbles of true vacuum is quenched.

As noted above however, the SM case is very far from the thin wall regime analyzed in [55], and before jumping to any conclusion, and also prior to studies that consider the inclusion of new physics, the stability analysis for the SM in the presence of gravity has to be performed. An early attempt to study the impact of gravity on the EW vacuum decay rate was done in [59], where a perturbative expansion of the bounce around the flat spacetime solution was considered. As later noted in [60] and then in [61] however, the boundary conditions for the bounce solution, that are essential in the calculation of the decay rate, are not respected already at the first order of the expansion, and the results of this work are at least questionable.

In order to get close to the SM case, but still keeping a simple model as in [55], a scalar theory with a potential whose parameters can be adjusted to explore cases far from the thin wall regime was considered in [60], and a numerical analysis of the false vacuum stability condition was performed. The main result is that for the potential that well approximates the SM case, the stabilizing effect of gravity is hardly seen even in very strong gravity regimes. As suggested in [62-64], the total quenching of the vacuum decay rate can eventually be reached at some very high scale. As shown in [60] however, for the SM case such an effect takes place in a far transplanckian regime where the theory has already lost its validity (similarly to what happens for the Landau pole in QED, where the latter occurs at such a high energy scale that the theory has lost its significance several orders of magnitudes below that scale). The results obtained with the simple model considered in [60] were later confirmed in [61], where a bona fide SM Higgs effective potential was used. 
In order to complete the stability analysis of the EW vacuum when gravity is taken into account, it is of the greatest importance to understand to which extent the presence of gravity can counteract the NP destabilizing effect discussed in [38-40, 43]. In the present work we address these issues, that are very important for current studies and for model building of BSM physics, where we are often confronted with the possibility of considering NP at Planckian and/or trans-Planckian scales. Anticipating on the results of the following sections, we will see that the tunneling time from the false to the true vacuum is still strongly dependent on NP even if it lives at very high ( $\left.\gg \phi_{\text {inst }}\right)$ scales, thus confirming the results of the analysis performed in the flat spacetime background [38-40, 43].

The rest of the paper is organized as follows. In section 2 the general theoretical set-up for our work, mainly consisting of the equations that will be used in the subsequent numerical analysis, is presented. Moreover, in order to keep the present paper as self-contained as possible, and also to check our tools against known results, the EW vacuum stability analysis in the absence of new physics in both flat and curved spacetime backgrounds is briefly sketched, and the known results are recovered. In section 3 we study the impact of $\mathrm{NP}$ at the Planck scale on the stability condition of the EW vacuum when the presence of gravity is taken into account, parametrizing NP in terms of higher order operators. In section 4 a different parametrization for NP at high energy scales is used, namely we introduce a new boson and a new fermion (with very large masses) coupled to the Higgs boson. Section 5 is for our conclusions.

\section{Theoretical background}

In the present section we briefly review the theoretical background for the computation of the tunneling decay rate from a Minkowski false vacuum (minimum of the potential with vanishing energy density) to an Anti-De Sitter (AdS) true vacuum (minimum of the potential with negative energy density), considering both the flat and the curved spacetime background cases.

Flat spacetime. Let us begin by considering the flat spacetime Euclidean action for a single component real scalar field $\phi$ :

$$
S[\phi]=\int d^{4} x\left[\frac{1}{2}\left(\partial_{\mu} \phi\right)^{2}+V(\phi)\right],
$$

where $V(\phi)$ is a potential with a local minimum (false vacuum) at $\phi=\phi_{\mathrm{fv}}$, and an absolute minimum (true vacuum) at $\phi=\phi_{\mathrm{tv}}$.

In order to calculate the false vacuum lifetime we have to look for the so called bounce solution to the Euclidean Euler-Lagrange equation that have $O(4)$ symmetry and satisfy certain boundary conditions $[53,54]$. If $r$ is the radial coordinate, the equation takes the form:

$$
\ddot{\phi}(r)+\frac{3}{r} \dot{\phi}(r)=\frac{d V}{d \phi},
$$

where the dot indicates derivative with respect to $r$, and the boundary condition are:

$$
\phi(\infty)=0 \quad \dot{\phi}(0)=0 .
$$


Denoting with $\phi_{\mathrm{b}}(r)$ the bounce solution, the action at $\phi_{\mathrm{b}}$ is:

$$
S\left[\phi_{\mathrm{b}}\right]=2 \pi^{2} \int_{0}^{\infty} d r r^{3}\left[\frac{1}{2} \dot{\phi}_{\mathrm{b}}^{2}+V\left(\phi_{\mathrm{b}}\right)\right]
$$

and the decay rate $\Gamma$ of the false vacuum is given by:

$$
\Gamma=D e^{-\left(S\left[\phi_{\mathrm{b}}\right]-S\left[\phi_{\mathrm{fv}}\right]\right)} \equiv D e^{-B}
$$

where $B \equiv S\left[\phi_{\mathrm{b}}\right]-S\left[\phi_{\mathrm{fv}}\right]$ is the so called tunneling exponent and the exponential of $-B$ gives the "tree-level" contribution to the decay rate, while $D$ is the quantum fluctuation determinant. If $V\left(\phi_{\mathrm{fv}}\right)=0$, the action $S\left[\phi_{\mathrm{fv}}\right]$ vanishes, and the tunneling exponent is simply $B=S\left[\phi_{\mathrm{b}}\right]$. In order to determine the false vacuum decay rate in the flat spacetime case, in the following we integrate numerically eq. (2.2) with boundary conditions (2.3), and use (2.4) to get the tunneling exponent $B$ of (2.5).

Curved spacetime. The next step is to study the impact of gravity on the vacuum decay rate, and to this end we consider the previous theory in a curved spacetime background. Including the Einstein-Hilbert term, the Euclidean action becomes:

$$
S\left[\phi, g_{\mu \nu}\right]=\int d^{4} x \sqrt{g}\left[-\frac{R}{16 \pi G}+\frac{1}{2} g^{\mu \nu} \partial_{\mu} \phi \partial_{\nu} \phi+V(\phi)\right]
$$

where $R$ is the Ricci scalar and $G$ is the Newton constant. Requiring again $O(4)$ symmetry, the (Euclidean) metric takes the form:

$$
d s^{2}=d r^{2}+\rho^{2}(r) d \Omega_{3}^{2}
$$

where $d \Omega_{3}^{2}$ is the unit 3-sphere line element and $\rho(r)$ is the volume radius of the 3 -sphere at fixed $r$ coordinate [55]. The bounce configuration needed to calculate the false vacuum transition rate is now given by the field and the metric solution, $\phi_{\mathrm{b}}(r)$ and $\rho_{\mathrm{b}}(r)$ respectively, of the coupled equations $(\kappa \equiv 8 \pi G)$ :

$$
\begin{aligned}
\ddot{\phi}+3 \frac{\dot{\rho}}{\rho} \dot{\phi} & =\frac{d V}{d \phi} \\
\dot{\rho}^{2} & =1+\frac{\kappa \rho^{2}}{3}\left(\frac{1}{2} \dot{\phi}^{2}-V(\phi)\right),
\end{aligned}
$$

where the first equation replaces (2.2), while the second is the only Einstein equation left by the symmetry. For the decay of a Minkowski false vacuum to a true AdS vacuum, the case of interest to us, the boundary conditions are:

$$
\phi_{\mathrm{b}}(\infty)=0 \quad \dot{\phi}_{\mathrm{b}}(0)=0 \quad \rho_{\mathrm{b}}(0)=0 .
$$

Asymptotically $(r \rightarrow \infty)$ the bounce $\phi_{\mathrm{b}}(r)$ approaches the constant false vacuum solution $\phi_{\mathrm{fv}}$, where $V\left(\phi_{\mathrm{fv}}\right)=0$ (Minkowski vacuum). In the same limit, from (2.8b) we see that the bounce solution metric $\rho_{\mathrm{b}}(r)$ approaches the flat spacetime metric:

$$
\rho_{\mathrm{b}}(r)=r+c .
$$


In the thin wall regime the constant $c$ is obtained analytically [55], while in general it is determined from the numerical integration of (2.8).

Differentiating (2.8b) with respect to $r$ we get:

$$
\ddot{\rho}=-\frac{\kappa}{3} \rho\left(\dot{\phi}^{2}+V(\phi)\right)
$$

that is a useful equation that we will use in our analysis as it is more robust than $(2.8 \mathrm{~b})$ for numerical integration [65]. Finally the Ricci scalar $R$ in terms of $\rho$ is given by:

$$
R=-\frac{6}{\rho^{2}}\left(\rho \ddot{\rho}+\dot{\rho}^{2}-1\right) .
$$

Inserting the above results in (2.6), the action for the bounce $\left(\phi_{\mathrm{b}}, \rho_{\mathrm{b}}\right)$ becomes:

$$
S_{\mathrm{b}} \equiv S\left[\phi_{\mathrm{b}}, \rho_{\mathrm{b}}\right]=2 \pi^{2} \int_{0}^{\infty} d r\left\{\rho_{\mathrm{b}}^{3}\left[\frac{1}{2} \dot{\phi}_{\mathrm{b}}^{2}+V\left(\phi_{\mathrm{b}}\right)\right]-\frac{3}{\kappa}\left(\rho_{\mathrm{b}} \dot{\rho}_{\mathrm{b}}^{2}+\rho_{\mathrm{b}}\right)\right\}+\left.\frac{6 \pi^{2}}{\kappa}\left(\rho_{\mathrm{b}}^{2} \dot{\rho}_{\mathrm{b}}\right)\right|_{0} ^{\infty} .
$$

As the false vacuum action $S\left[\phi_{\mathrm{fv}}, \rho_{\mathrm{fv}}\right]$ contains the same boundary term (the last one) of (2.13), in the tunneling exponent $B$ this term does not appear. Neglecting it, and using (2.8) in (2.13) we finally get:

$$
S_{\mathrm{b}}=4 \pi^{2} \int_{0}^{\infty} d r\left[\rho_{\mathrm{b}}^{3} V\left(\phi_{\mathrm{b}}\right)-\frac{3}{\kappa} \rho_{\mathrm{b}}\right] .
$$

From (2.14), the false vacuum action $S_{\mathrm{fv}} \equiv S\left[\phi_{\mathrm{fv}}, \rho_{\mathrm{fv}}\right]$ can be easily derived. As already said, we are interested in the decay of a Minkowski false vacuum (the state that corresponds to the minimum of the potential with vanishing energy density, $\left.V\left(\phi_{\mathrm{fv}}\right)=0\right)$ to an AdS true vacuum. For the false vacuum case, $\phi(r)=\phi_{\mathrm{fv}}$, and $\dot{\rho}^{2}=1$, i.e. $\rho_{\mathrm{fv}}(r)=r+$ const. This latter constant is fixed and is $c$ of eq. (2.10), as the bounce metric asymptotically tends to the flat spacetime metric of the Minkowski false vacuum. In calculating the action $S_{\mathrm{fv}}$ we must then integrate over the Minkowski spacetime from a real radius equal to zero up to infinity. This corresponds to the integration range $[-c, \infty)$ in the $r$ coordinate,

$$
S_{\mathrm{fv}}=-4 \pi^{2} \int_{-c}^{\infty} d r \frac{3}{\kappa}(r+c)=-4 \pi^{2} \int_{0}^{\infty} d r \frac{3}{\kappa}(r+c)-\frac{6 \pi^{2}}{\kappa} c^{2},
$$

so that for the tunneling exponent $B=S_{\mathrm{b}}-S_{\mathrm{fv}}$ we have:

$$
B=4 \pi^{2} \int_{0}^{\infty} d r\left[\rho^{3} V\left(\phi_{\mathrm{b}}\right)-\frac{3}{\kappa}(\rho-r-c)\right]+\frac{6 \pi^{2}}{\kappa} c^{2} .
$$

In the following, the decay rate of the Minkowski false vacuum into the AdS vacuum will be calculated by integrating numerically eqs. (2.8a) and (2.11) with boundary conditions (2.9). Then with the help of (2.16) the tunneling exponent will be obtained.

As we are interested in the stability analysis of the EW vacuum, in our case the scalar field $\phi$ is the Higgs field, and the potential $V(\phi)$ is the Higgs effective potential. More specifically it is the renormalization group improved potential, that can be written as:

$$
V_{\mathrm{SM}}(\phi) \sim \frac{1}{4} \lambda_{\mathrm{SM}}(\phi) \phi^{4},
$$


where $\lambda_{\mathrm{SM}}(\phi)$ is the quartic running coupling $\lambda_{\mathrm{SM}}(\mu)$ ( $\mu$ is the running scale) with $\mu=$ $\phi[2,66]$.

In order to get $\lambda_{\mathrm{SM}}(\mu)$, the system of RG equation of the SM couplings has to be run. In [61], where a stability analysis of the SM in the presence of gravity was performed, an improved Higgs potential obtained with the help of three-loops beta functions was used, while in previous analyses two-loop beta functions were considered [18]. In this respect, we note that the consistent counting of loops in the beta functions of different SM particles poses a problem in itself, as it was found that taking the same order for all the different components of the SM (Yukawa, gauge and quartic couplings) actually leads to inconsistencies [67].

The purpose of the present work however is to study the impact that NP at high energies can have on the stability condition of the EW vacuum when the SM coupling to gravity is taken into account. We are then not interested in precision measurements and/or refinements of previous analyses. For our illustrative scopes, the differences between these cases (the two loops counting, the three loops counting, and the "consistent" counting proposed in [67]) are minimal, and they have no impact on the results and conclusions of our analysis.

We can then leave aside these questions and work in a simplified yet very robust framework, by using a good approximation of the SM effective potential that was obtained in [68] by fitting the two-loops improved Higgs potential with the three parameter function [68]:

$$
\lambda_{\mathrm{SM}}(\phi)=\lambda_{*}+\alpha\left(\ln \frac{\phi}{M_{\mathrm{P}}}\right)^{2}+\beta\left(\ln \frac{\phi}{M_{\mathrm{P}}}\right)^{4},
$$

where $M_{\mathrm{P}}=1 / \sqrt{G}$ is the Planck mass. The fit gives:

$$
\lambda_{*}=-0.013 \quad \alpha=1.4 \times 10^{-5} \quad \beta=6.3 \times 10^{-8} .
$$

In the following we work with the Higgs potential (2.17) with $\lambda_{\mathrm{SM}}(\phi)$ given by $(2.18)$ and (2.19).

Both in the flat and curved spacetime cases, an important parameter is the size $\mathcal{R}$ of the bounce, defined as the value of $r$ such that

$$
\phi_{\mathrm{b}}(\mathcal{R})=\frac{1}{2} \phi_{\mathrm{b}}(0)
$$

Going back to (2.5) for the vacuum decay rate, we note that a good approximation to the prefactor for the case that we are considering is given in terms of the bounce size $\mathcal{R}$ and of $T_{\mathrm{U}}$, the age of the Universe, and the EW vacuum tunneling time $\tau=\Gamma^{-1}$ turns out to be [40]:

$$
\tau \simeq\left(\frac{\mathcal{R}^{4}}{T_{\mathrm{U}}^{3}}\right) e^{B}
$$

In the following we use (2.21) to calculate the false vacuum lifetime.

Before ending this section and moving to the study of the impact of NP on the EW vacuum stability, we would like to test our tools starting with the known cases of the flat and curved spacetime backgrounds in the absence of NP (i.e. considering the SM alone), and briefly sketch the analysis for these cases. 
Flat spacetime. In order to proceed with the numerical solution of the bounce equation (2.2), we begin by scaling the dimensionful field $\phi$ and the radial coordinate $r$ to dimensionless quantities, $x$ and $\varphi(x)$ respectively, by using Planck units:

$$
x \equiv M_{\mathrm{P}} r \quad \varphi(x) \equiv \frac{\phi(r)}{M_{\mathrm{P}}} .
$$

Eq. (2.2), the boundary conditions (2.3) and the potential (2.17) then become:

$$
\begin{aligned}
\varphi^{\prime \prime}(x)+\frac{3}{x} \varphi^{\prime}(x) & =\frac{d U}{d \varphi} \\
\varphi(\infty) & =0 \quad \varphi^{\prime}(0)=0 \\
U(\varphi) & =\frac{1}{4} \varphi^{4}\left(\lambda_{*}+\alpha \ln ^{2} \varphi+\beta \ln ^{4} \varphi\right),
\end{aligned}
$$

where the prime indicates the derivative respect to $x$. After the rescaling (2.22), the tunneling exponent (2.4) becomes:

$$
B=2 \pi^{2} \int_{0}^{\infty} d x x^{3}\left[\frac{1}{2} \varphi_{\mathrm{b}}^{\prime}(x)+U\left(\varphi_{\mathrm{b}}\right)\right] .
$$

Solving numerically the bounce equation (2.23), with the Higgs potential given by (2.25) and (2.19), and inserting the result for $\varphi_{\mathrm{b}}(x)$ in (2.26), after using the values found for $B$ and $\mathcal{R}$, namely $B=2025.27$ and $\mathcal{R}=10.7597$, we finally get for the lifetime $\tau$ of the EW vacuum:

$$
\tau_{\text {flat }} \sim 10^{639} T_{\mathrm{U}},
$$

in very good agreement with the results known in the literature. This is the first test of our numerical method, and also shows that we are considering a good approximation for the Higgs potential.

Curved spacetime. As in the case of flat spacetime, we move to dimensionless quantities. Defining the dimensionless curvature $a(x)=M_{\mathrm{P}} \rho(r)$, eqs. (2.8a) and (2.11) (that will be used in the following numerical integration) become:

$$
\begin{aligned}
\varphi^{\prime \prime}+3 \frac{a^{\prime}}{a} \varphi^{\prime} & =\frac{d U}{d \varphi} \\
a^{\prime \prime} & =-\frac{8 \pi}{3} a\left(\varphi^{\prime 2}+U\right),
\end{aligned}
$$

where the potential $U(\varphi)$ is the same as in (2.25). The corresponding boundary conditions are:

$$
\varphi(\infty)=0 \quad \varphi^{\prime}(0)=0 \quad a(0)=0 \quad a^{\prime}(0)=1 .
$$

As we have already said, $\rho(r) \sim r$ for $r \rightarrow \infty$, and the asymptotic $(x \rightarrow \infty)$ behavior of the bounce solution in the presence of gravity is the same as in the flat spacetime case.

In terms of dimensionless quantities, from (2.16) we find for tunneling exponent:

$$
B=4 \pi^{2} \int_{0}^{\infty} d x\left[a_{\mathrm{b}}^{3} U\left(\varphi_{\mathrm{b}}\right)-\frac{3}{8 \pi}\left(a_{\mathrm{b}}-x-c\right)\right]+\frac{3 \pi}{4} c^{2}
$$



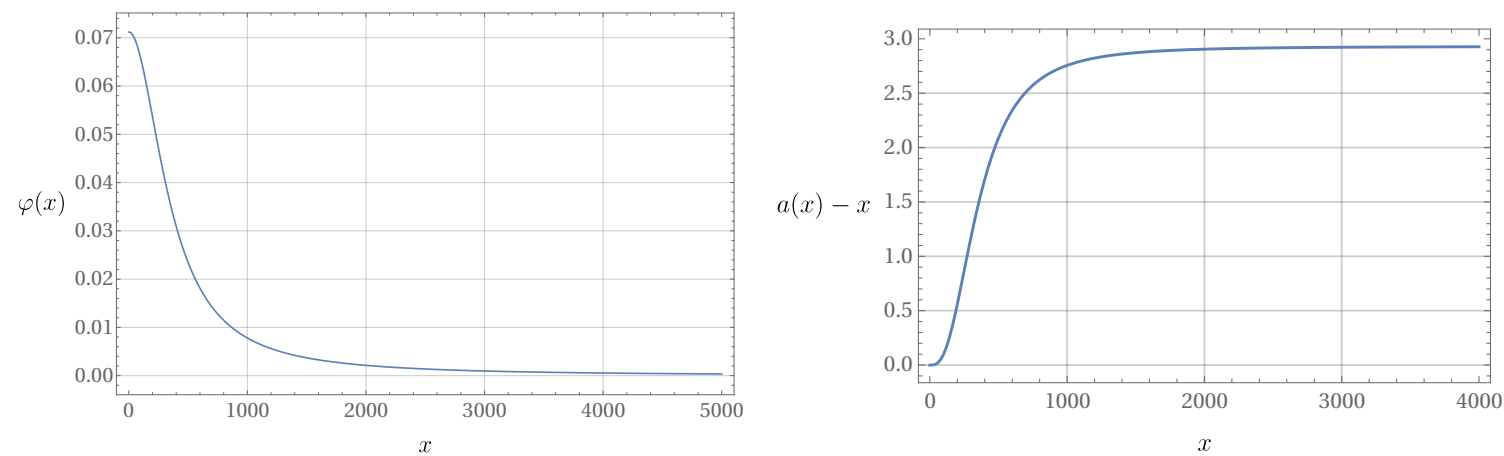

Figure 1. Left panel: profile of the bounce solution $\varphi(x)$ in the presence of gravity. It is obtained for the potential (2.25) with the parameters $\lambda_{*}, \alpha, \beta$ given in (2.19). The center of the bounce is at $\varphi(0)=0.0712$, its size is $\mathcal{R}=350.2996$ and the tunneling exponent is $B=2062.5836$. Right panel: difference between the curvature radius and its asymptotic value, $a(x)-x$, for the same parameters as in the left panel.

where $c$ (as already said above) is the constant that determines the asymptotic behavior of the metric $a(x)$ for $x \rightarrow \infty$, while $\left(\varphi_{\mathrm{b}}, a_{\mathrm{b}}\right)$ is the bounce solution to the system (2.28).

In the left panel of figure 1 the bounce profile $\varphi_{\mathrm{b}}(x)$ is plotted. The right panel shows the difference $a_{\mathrm{b}}(x)-x$ : we clearly see how asymptotically $a_{\mathrm{b}}(x)$ reaches the Minkowskian behavior $a(x) \sim x+c$, and we can read the value of the constant $c$. Finally, with the help of (2.21), we obtain the tunneling time in the presence of gravity:

$$
\tau_{\text {grav }} \sim 10^{661} T_{\mathrm{U}} .
$$

Once again we observe that the above result is in good agreement with known results [61]. Moreover, comparing (2.31) with the corresponding flat spacetime tunneling time (2.27), we see that gravity (as expected) tends to stabilize the EW vacuum.

\section{New physics: higher order operators}

The results briefly presented in section 2 are known and concern the stability analysis under the assumption that, in the event that unknown NP lives close to $M_{\mathrm{P}}$, it has no impact on the stability condition of the EW vacuum. In other words it is assumed that new physics at $M_{\mathrm{P}}$ is decoupled from the physics that triggers the EW vacuum decay, and that it should be possible to calculate the tunneling rate ignoring these terms.

The analysis of the previous section is essential to set the proper framework where the effects of the presence of NP at $M_{\mathrm{P}}$ can be properly investigated. We parametrize NP as in [38-40] with the help of higher powers of $\phi$ added to the Higgs potential:

$$
V_{\mathrm{NP}}(\phi)=\frac{\lambda_{6}}{6} \frac{\phi^{6}}{M_{\mathrm{P}}^{2}}+\frac{\lambda_{8}}{8} \frac{\phi^{8}}{M_{\mathrm{P}}^{4}} .
$$

It was shown in [38-40] for the flat spacetime case that when $\lambda_{6}<0$ and $\lambda_{8}>0$ the potential (3.1) destabilizes the EW vacuum. In other words, these NP terms favor the 


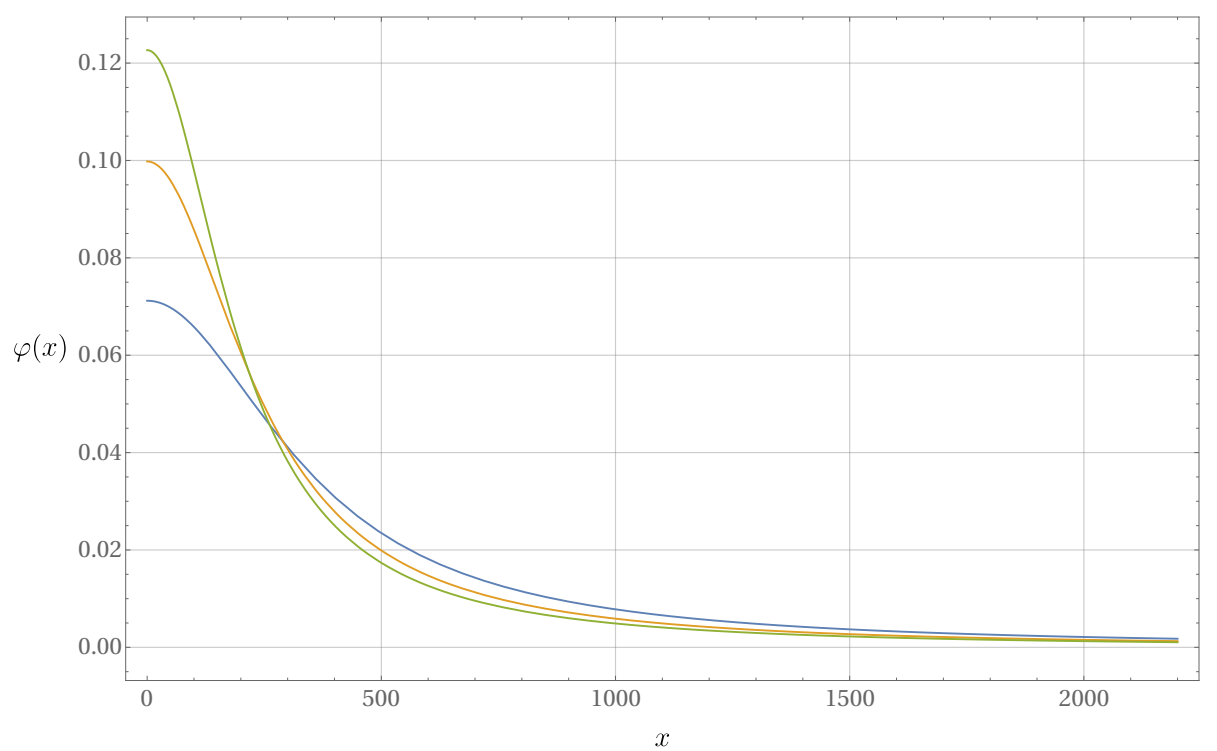

Figure 2. The blue curve is the profile of the bounce solution obtained for the potential (3.2) with $\lambda_{6}=0$ and $\lambda_{8}=0$, i.e. in the absence of new physics. The yellow curve is the profile of the bounce solution for $\lambda_{6}=-0.03$ and $\lambda_{8}=0.03$, while the green one is the profile of the bounce obtained for $\lambda_{6}=-0.04$ and $\lambda_{8}=0.04$. Note that with increasing values of the couplings the center of the bounce $\varphi(0)$ becomes larger while the size diminishes.

nucleation of true vacuum bubbles and, depending on the specific values of $\lambda_{6}$ and $\lambda_{8}$, this destabilization effect could dramatically reduce the EW vacuum lifetime $\tau$ in (2.27) and make it even shorter than the age of the Universe $T_{\mathrm{U}}$. We now consider the same kind of analysis in the presence of gravity.

Adding the NP terms (3.1) to the SM Higgs potential (2.17), and moving again to dimensionless quantities, the new dimensionless potential $U(\varphi)$ becomes:

$$
U(\varphi)=\frac{1}{4} \varphi^{4}\left(\lambda_{*}+\alpha \ln ^{2} \varphi+\beta \ln ^{4} \varphi+\frac{2}{3} \lambda_{6} \varphi^{2}+\frac{1}{2} \lambda_{8} \varphi^{4}\right) .
$$

We are now ready to study the impact of high energy NP on the EW vacuum stability condition in the presence of gravity.

A first important result of our analysis is that for each value of the couple $\left(\lambda_{6}, \lambda_{8}\right)$ there is a different bounce solution to eqs. (2.28), all of them being different from the solution obtained for the SM alone, i.e. the case $\lambda_{6}=0, \lambda_{8}=0$. Therefore, the "new" bounce solutions related to the presence of new physics, here parametrized in terms a given couple $\left(\lambda_{6}, \lambda_{8}\right)$, is still present even when in the stability analysis gravity is explicitly taken into account.

In order to illustrate these results, in figure 2 we show bounce solutions to eqs. (2.28) for $\lambda_{6}=-0.03, \lambda_{8}=0.03$ (yellow curve), $\lambda_{6}=-0.04, \lambda_{8}=0.04$ (green curve) and compare them with the corresponding $\lambda_{6}=0, \lambda_{8}=0$ (blue curve) case. The profiles obtained are definitely new solutions to these equations related to the specific values of $\lambda_{6}$ and $\lambda_{8}$, clearly different from the bounce (blue curve) obtained for the SM alone $\left(\lambda_{6}=0\right.$ and $\left.\lambda_{8}=0\right)$. 


\begin{tabular}{|cc|cc|}
\hline$\lambda_{6}$ & $\lambda_{8}$ & $\tau_{\text {flat }} / T_{\mathrm{U}}$ & $\tau_{\text {grav }} / T_{\mathrm{U}}$ \\
\hline 0 & 0 & $10^{639}$ & $10^{661}$ \\
\hline-0.05 & 0.1 & $10^{446}$ & $10^{653}$ \\
\hline-0.1 & 0.2 & $10^{317}$ & $10^{598}$ \\
\hline-0.15 & 0.25 & $10^{186}$ & $10^{512}$ \\
\hline-0.3 & 0.3 & $10^{-52}$ & $10^{287}$ \\
\hline-0.45 & 0.5 & $10^{-93}$ & $10^{173}$ \\
\hline-0.7 & 0.6 & $10^{-162}$ & $10^{47}$ \\
\hline-1.2 & 1.0 & $10^{-195}$ & $10^{-58}$ \\
\hline-1.7 & 1.5 & $10^{-206}$ & $10^{-106}$ \\
\hline-2.0 & 2.1 & $10^{-206}$ & $10^{-121}$ \\
\hline
\end{tabular}

Table 1. Tunneling time for different values of $\lambda_{6}$ and $\lambda_{8}$, both for the flat and curved spacetime cases. We note that although gravity tends to stabilize the EW vacuum (the tunneling time $\tau_{\text {grav }}$ is always higher than the corresponding one in flat spacetime $\left.\tau_{\text {flat }}\right)$, new physics has always a strong impact.

With the help of (2.21) we now calculate the EW vacuum lifetime for different values of the NP couplings $\lambda_{6}$ and $\lambda_{8}$. The fourth column of table 1 contains different values of the tunneling time obtained for different couples $\left(\lambda_{6}, \lambda_{8}\right)$. For comparison, the third column contains the corresponding values of $\tau$ for the flat spacetime analysis. First of all we note that the effect already seen in the previous section (also reported in the first line of the table, the case $\lambda_{6}=0, \lambda_{8}=0$ ), namely that the presence of gravity tends to stabilize the EW vacuum, is maintained even in the presence of new physics.

However, a simple inspection of this table shows that even though the presence of gravity tends to stabilize the EW vacuum as compared to the corresponding flat spacetime case, still for $O(1)$ values of the new physics couplings $\lambda_{6}$ and $\lambda_{8}$ the tunneling time can be made smaller than the age of the Universe $T_{\mathrm{U}}$. Let us consider just a couple of examples. For $\lambda_{6}=-0.3$ and $\lambda_{8}=0.3$ for instance, the EW vacuum in the flat spacetime background is unstable, being $\tau \sim 10^{-52} T_{\mathrm{U}}$, but for the corresponding case with gravity included we observe a stabilization of the EW vacuum: $\tau \sim 10^{287} T_{\mathrm{U}}$. There is a competition between the destabilizing effect of NP and the stabilizing effect of gravity. In this example, gravity takes over new physics and as a result the EW vacuum turns out to be stable. However for larger (absolute) values of the NP couplings, the destabilizing effect of NP takes over the stabilizing effect of gravity. For instance, for $\lambda_{6}=-1.2$ and $\lambda_{8}=1.0$, despite the stabilizing effect of gravity $\left(\tau_{\text {grav }} \gg \tau_{\text {flat }}\right.$ ), the EW vacuum turns out to be unstable: $\tau_{\text {grav }} \sim 10^{-58} T_{\mathrm{U}}$.

The results discussed above with the help of table 1 are better summarized in figure 3 where the stability diagram in the $\left(\lambda_{6}, \lambda_{8}\right)$ plane is presented for the range of values $-1.5<$ $\lambda_{6}<-0.4$ and $0.4<\lambda_{8}<1.5$.

We see that, when the analysis is performed in the flat spacetime case (i.e. when we ignore the presence of gravity), the stability region $\left(\tau>T_{\mathrm{U}}\right.$, blue area) is confined to the 


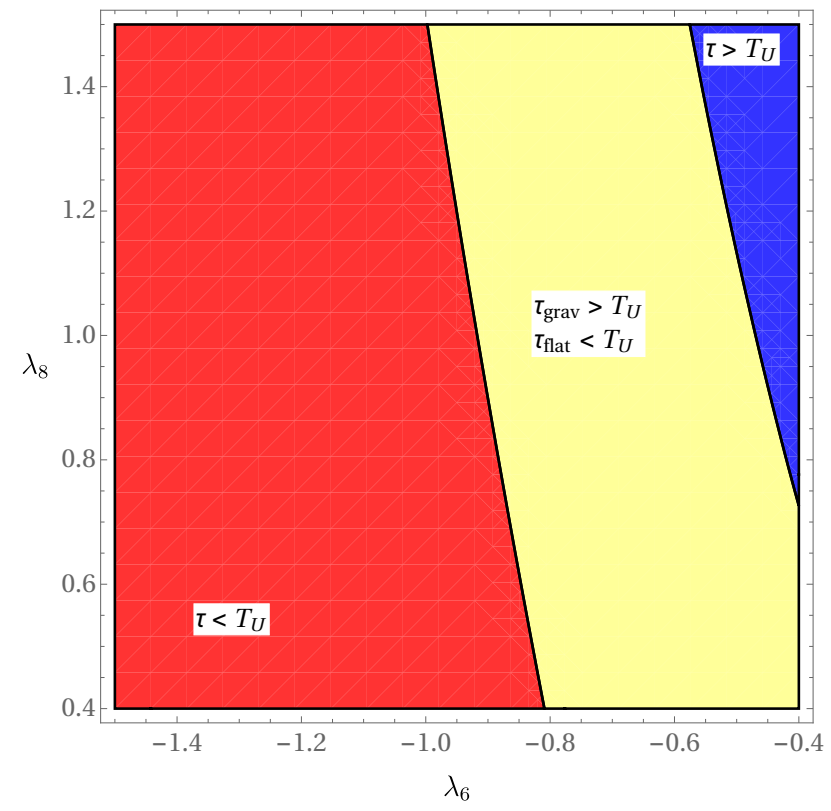

Figure 3. Stability diagram in the $\left(\lambda_{6}, \lambda_{8}\right)$ plane showing three separate regions where: (i) $\tau_{\text {flat }}, \tau_{\text {grav }}>T_{\mathrm{U}}$ (blue region); (ii) $\tau_{\text {flat }}<T_{\mathrm{U}}, \tau_{\text {grav }}>T_{\mathrm{U}}$ (yellow region); (iii) $\tau_{\text {flat }}, \tau_{\text {grav }}<T_{\mathrm{U}}$ (red region). Note that, as in the blue (red) area $\tau_{\text {flat }}$ and $\tau_{\text {grav }}$ are both larger (smaller) than $T_{\mathrm{U}}$, in these regions we wrote $\tau>T_{\mathrm{U}}\left(\tau<T_{\mathrm{U}}\right)$ with no further specification. The potential of (2.25) and parameters $\lambda_{*}, \alpha, \beta$ given in (2.19) are used. Note that although the inclusion of gravity induces an enlargement of the region with EW vacuum lifetime larger than $T_{\mathrm{U}}$, for a large portion of the phase diagram we have $\tau<T_{\mathrm{U}}$, even when gravity is taken into account.

upper right corner of this figure. When the presence of gravity is taken into account, the stability region $\left(\tau>T_{\mathrm{U}}\right)$ broadens (blue + yellow area), and this shows the tendency of gravity toward stabilization. Yet, for a large portion of the parameter space the EW vacuum is still unstable, thus showing that the potential stabilization that should be induced by gravity is not sufficient to counteract against the destabilization mechanism due to the presence of high energy NP. In this respect, it is important to note that this destabilization occurs for physical $O(1)$ values of the coupling constants $\lambda_{6}$ and $\lambda_{8}$.

In figure 4 we show some more quantitative details of the phase diagram drawn in figure 3, presenting the flat spacetime case (left panel) separately from the case when gravity is taken into account (right panel). In this figure the black lines are level curves on which the relative constant value of $\log _{10}\left(\tau / T_{\mathrm{U}}\right)$ is reported. The thick red dashed lines indicate the points where $\tau=T_{\mathrm{U}}$.

Let us summarize the results of the present section. We have seen that the stability condition of the EW vacuum is the result of a competition between the destabilizing effect of NP and the stabilizing tendency of gravity. However, even keeping the values of the coupling constants in the natural $O(1)$ range, for a large portion of the parameter space the destabilization induced by Planckian NP largely overwhelms the tendency of gravity toward stabilizing the EW vacuum: the contribution to the decay rate through the new bounce 

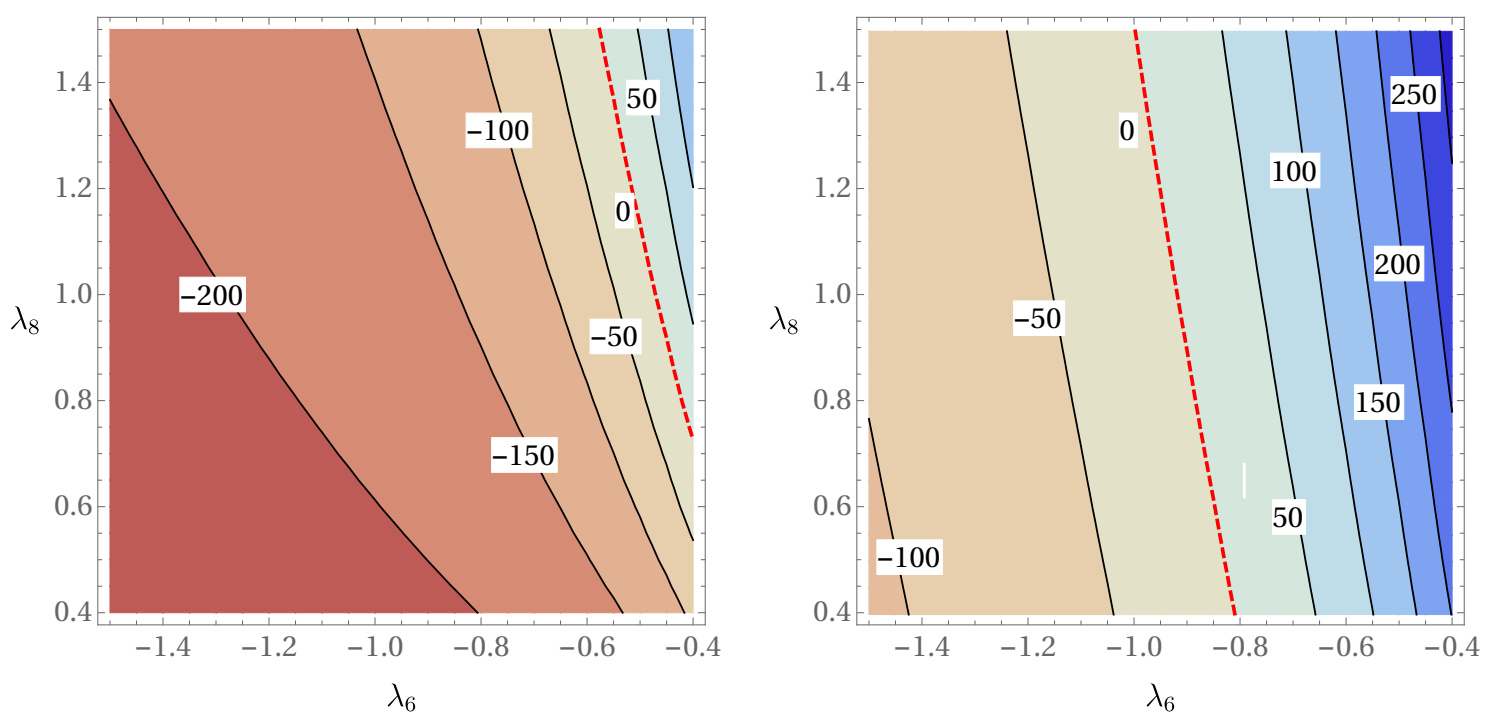

Figure 4. Stability diagrams in the $\left(\lambda_{6}, \lambda_{8}\right)$ plane for $\log _{10}\left(\tau / T_{\mathrm{U}}\right)$ with the potential of $(2.25)$ and parameters $\lambda_{*}, \alpha, \beta$ given in (2.19). Left panel: flat spacetime case. Right panel: curved spacetime case. In each of the two panels, the black lines are curves with fixed values of $\log _{10}\left(\tau / T_{\mathrm{U}}\right.$ ) (reported on it), while the thick red dashed line indicates the points where $\tau=T_{\mathrm{U}}$. Colors serve as a guidance, indicating the decreasing of $\log _{10}\left(\tau / T_{\mathrm{U}}\right)$ from the right to the left. Note that the inclusion of gravity induces a broad enlargement of the region where the EW vacuum is stable $\left(\log _{10}\left(\tau / T_{\mathrm{U}}\right)>0\right)$.

solution by far dominates the contribution coming from the bounce solution obtained by considering SM terms only.

\section{New physics: fermions and bosons with large masses}

In the present section the stability analysis of the EW vacuum will be performed by considering a different parametrization for NP at high energy scales. Actually in [38-40] and in the previous section the analysis was performed by parametrizing NP at the Planck scale in terms of few higher order (non-renormalizable) operators. This is just a convenient and efficient way of mimicking the presence of new physics, clearly not an (illegitimate) truncation of the UV completion of the SM. Some authors however expressed a certain skepticism on these results, suggesting that this effect should disappear when the infinite tower higher dimensional operators of the renormalizable UV completion of the SM is taken into account, so that the expected decoupling of very high energy physics from the mechanism that triggers the decay of the false vacuum should be recovered. It was actually suspected that this effect takes place above the physical cutoff, where the control of the theory is lost [69].

Although it is understandable that the parametrization of NP in terms of higher order operators could be the source of a certain confusion, the destabilizing effect has nothing to do with this parametrization. For the case of a flat spacetime background in [43] the stability analysis was performed by parametrizing NP in terms of renormalizable additional 
terms, with a fermion and a boson with very high masses that interact with the Higgs field, and it was shown that the destabilizing effect found in [38-40] is still present.

In this section we present the same kind of analysis of [43] taking into account the presence of gravity (i.e. considering the case of a curved spacetime background), and show that as for the case of the parametrization used in the previous section, gravity does not produce any washing out of the destabilizing effect of new physics, although it slightly mitigates it.

In order to illustrate the destabilization phenomenon we consider as in [43] a renormalizable model that is not a realistic high energy UV-completion of the SM but is very appropriate to the purposes of the present work. NP lives at very high energy scales and is parametrized by adding to the SM a scalar field $S$ and a fermion field $\psi$ that interact in a simple way with the Higgs field $\phi$, with very large masses (see below) $M_{S}$ and $M_{f}$ of the scalar and fermion respectively.

Apart from the kinetic terms, the additional terms in the Lagrangian are:

$$
\Delta \mathcal{L}=\frac{M_{S}^{2}}{2} S^{2}+\frac{\lambda_{S}}{4} S^{4}+g_{S} \phi^{2} S^{2}+M_{f} \bar{\psi} \psi+g_{f} \phi \bar{\psi} \psi
$$

To understand how a NP Lagrangian of this kind can arise in a physical setup, we note that the large mass term $M_{f}$ can be thought as a sort of heavy right handed "neutrino" in the framework of a see-saw mechanism. While the corresponding light "neutrino" is totally harmless for the stability of the EW vacuum, the heavy "neutrino" can play an important role in destabilizing the vacuum. The scalar field $S$ counterbalances the destabilizing effect of $\psi$. Note that models with new scalar fields coupled to the Higgs (although admittedly unrealistic) have already been used to provide a stabilization mechanism for the Higgs effective potential [70, 71].

Before proceeding with our work, it is worth to mention some issues related to the gauge dependence/invariance of physical quantities involved in the stability analysis. Let us start by recalling that the effective potential away from the extrema is gauge dependent [45-47]. In particular, absolute stability bounds on the Higgs mass (formally gauge independent) turn out to be gauge dependent at any order of perturbation theory, and only when a consistent resummation is considered the result is made gauge independent, providing a slight improvement in mass bounds [47]. The gauge dependence of the instability scale has also been investigated and the range of uncertainty identified [46], but it is not known if it is possible to calculate this quantity in a gauge independent manner.

Turning now to the main quantity of interest to us, namely the false vacuum decay rate, we know that it is a gauge-invariant quantity. Since very recently, however, it was not known how to implement an explicit gauge-invariant calculation of this quantity. The prescription for such a computation was finally given in [48]. Successively, in $[49,50]$ the results of the latter analysis were applied to the computation of the EW vacuum decay rate in the SM.

Let us continue now with our analysis. For the purposes of the present work, it is sufficient to consider the impact of the additional terms (4.1) on the Higgs effective potential $V(\phi)$ at the one-loop level only. In fact we do not need a better level of precision as we 
are not interested in extracting numbers but we only want to illustrate the destabilization effect that arises from very high energy physics (see also the considerations developed below eq. (2.17)). The one-loop contribution to $V(\phi)$ from these terms is:

$$
\begin{aligned}
V_{1}(\phi)= & \frac{\left(M_{S}^{2}+2 g_{S} \phi^{2}\right)^{2}}{64 \pi^{2}}\left[\ln \left(\frac{M_{S}^{2}+2 g_{S} \phi^{2}}{M_{S}^{2}}\right)-\frac{3}{2}\right] \\
& -\frac{\left(M_{f}^{2}+g_{f}^{2} \phi^{2}\right)^{2}}{16 \pi^{2}}\left[\ln \left(\frac{M_{f}^{2}+g_{f}^{2} \phi^{2}}{M_{S}^{2}}\right)-\frac{3}{2}\right],
\end{aligned}
$$

where the renormalization scale $\mu$ is taken as $\mu=M_{S}$. In this respect we note that at very high values of the running scale the SM quartic coupling reaches a plateau: $\lambda_{\mathrm{SM}}(\mu)$ has practically the same value in the whole range $\left[M_{f}, M_{S}\right]$, and this is why we can use $\lambda_{\mathrm{SM}}\left(M_{S}\right)$ as the threshold value for the coupling (even though strictly speaking we should use $\lambda_{\mathrm{SM}}\left(M_{f}\right)$ (see below)), and can choose $\mu=M_{S}$ as the renormalization (threshold) scale.

The presence of the high energy NP of (4.1) is then taken into account by adding to the SM potential $V_{\mathrm{SM}}(\phi)$ in (2.17) and (2.18) the contribution coming from $V_{1}(\phi)$. To this end we have to implement the matching conditions described below. First of all we expand the potential $V_{1}(\phi)$ in powers of $\phi$ and isolate the constant, the $\phi^{2}$ and the $\phi^{4}$ terms. Then at the threshold scale $M_{f}$ we require that: (i) the renormalized cosmological constant $\Lambda$, given by the sum of all the constant terms (those coming from the SM potential and those coming from $\left.V_{1}(\phi)\right)$ vanishes, $\Lambda\left(\mu=M_{f}\right) \sim 0$; (ii) the renormalized mass term, given by the sum of all the coefficients of $\phi^{2}$, and identified with the SM mass parameter $m_{\mathrm{SM}}^{2}\left(\mu=M_{f}\right)$ at the scale $M_{f}$, vanishes: $m_{\mathrm{SM}}^{2}\left(\mu=M_{f}\right) \sim 0$ (more precisely we neglect this term to a very high degree of accuracy for the large values of $\phi$ considered); (iii) the renormalized quartic coupling, given by the sum of all the coefficients of $\phi^{4}$, is identified with the SM quartic coupling at the scale $M_{f}, \lambda_{\mathrm{SM}}\left(\mu=M_{f}\right)$. In other words, at the scale $M_{f}$ this coefficient is matched with the value of the quartic coupling obtained by considering the running of the renormalization group equations for the SM couplings alone.

The above requirements for the renormalized cosmological constant and mass are well known features. For the renormalized $\Lambda$ (apart from the fine tuning problem) we can practically consider that $\Lambda(\mu=0) \sim \Lambda\left(\mu=M_{f}\right) \sim 0$. The same is true for the renormalized mass, for which we take $m^{2}(\mu=0) \sim m^{2}\left(\mu=M_{f}\right) \sim 0$, meaning that we neglect the $\phi^{2}$ term as compared to the $\phi^{4}$ and other terms for these large values of $\phi$, and that the running of the renormalized mass is totally harmless in this respect. For the quartic coupling we have a true matching condition. In fact we require that at the threshold scale $\mu=M_{f}$ the quartic coupling coincides with $\lambda_{\mathrm{SM}}\left(\mu=M_{f}\right)$, that is obtained by running the renormalization group equations for the SM couplings only. Practically starting from the scale $M_{f}$, the potential is given by the SM contribution $V_{\mathrm{SM}}(\phi)$ plus the contribution of $V_{1}(\phi)$ subtracted of its constant, quadratic and quartic powers of $\phi$, that we call $\bar{V}_{1}(\phi)$ from now on:

$$
V_{\mathrm{tot}}(\phi)=\frac{1}{4} \lambda_{\mathrm{SM}}(\phi) \phi^{4}+\bar{V}_{1}(\phi)
$$



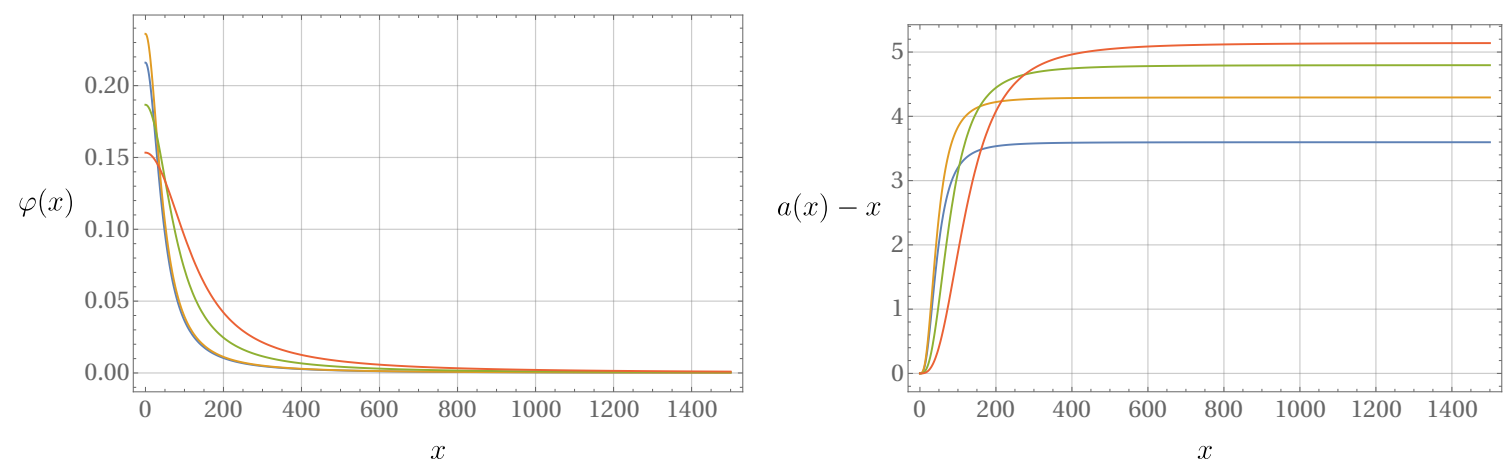

Figure 5. Left panel: profile of the bounce solutions $\varphi(x)$ for the potential (4.3) relative to the four cases considered in the text: $M_{S}=2.5 \times 10^{-1}, M_{f}=3 \times 10^{-4}, g_{S}=0.96, g_{f}^{2}=0.5$ (yellow); $M_{S}=2.0 \times 10^{-1}, M_{f}=10^{-4}, g_{S}=0.9, g_{f}^{2}=0.5$ (blue); $M_{S}=2.0 \times 10^{-1}, M_{f}=10^{-3}, g_{S}=0.95$, $g_{f}^{2}=0.4$ (green); $M_{S}=1.5 \times 10^{-1}, M_{f}=5 \times 10^{-3}, g_{S}=0.92, g_{f}^{2}=0.4$ (red). Right panel: the corresponding difference between the curvature radius and its asymptotic value, $a(x)-x$, for the same parameters as in the left panel.

We are now ready to use our model of high energy NP to calculate the EW vacuum lifetime for different values of the masses $M_{f}$ and $M_{S}$ of $\psi$ and $S$, and for different values of the coupling constants. For our illustrative purposes we have chosen to consider the four following examples: (i) $M_{S}=2.5 \times 10^{-1}, M_{f}=3 \times 10^{-4}, g_{S}=0.96, g_{f}^{2}=0.5$; (ii) $M_{S}=2.0 \times 10^{-1}, M_{f}=10^{-4}, g_{S}=0.9, g_{f}^{2}=0.5$; (iii) $M_{S}=2.0 \times 10^{-1}, M_{f}=10^{-3}$, $g_{S}=0.95, g_{f}^{2}=0.4$; (iv) $M_{S}=1.5 \times 10^{-1}, M_{f}=5 \times 10^{-3}, g_{S}=0.92, g_{f}^{2}=0.4$.

First of all we have to solve the bounce equations (2.28) for $\varphi(x)$ and $a(x)$. In figure 5 the profiles of the bounce solutions $\varphi_{\mathrm{b}}(x)$ for the four different cases (i), (ii), (iii) and (iv) and the corresponding plots of $a_{\mathrm{b}}(x)-x$ are presented, with colors yellow, blue, green and red respectively.

These are the first relevant results of the present section: in the presence of NP at very high energies, new bounce solutions exist not only when the analysis is carried in the flat spacetime background [43] but also when we take into account the presence of gravity. These results reinforce those of the previous section, where high energy NP was parametrized in terms of higher order operators, and show that the appearance of new bounce solutions is not an artifact of the specific parametrization used in section 3 .

Using (2.21) to calculate the vacuum lifetime, for the examples considered above we find in units of $T_{\mathrm{U}}$ (going from (i) to (iv)):

$$
\tau=10^{-65}, \quad \tau=10^{-93}, \quad \tau=10^{94}, \quad \tau=10^{307},
$$

to be compared with the corresponding results for the tunneling time obtained from the analysis performed in a flat spacetime background, where we have:

$$
\tau=10^{-80}, \quad \tau=10^{-103}, \quad \tau=10^{80}, \quad \tau=10^{293} .
$$

Eqs. (4.4) and (4.5) together with figure 5 contain the main lesson of the present section. They definitely show that, even when gravity is included in the analysis, the presence of 
$\mathrm{NP}$ at high energy scales can have an enormous impact on the vacuum lifetime. It is worth to remind here that when the calculation is performed in the curved spacetime background and the presence of high energy new physics is not considered, the tunneling time is given by $(2.31)\left(\tau \sim 10^{661} T_{\mathrm{U}}\right)$, while from (4.4) we see that $\tau$ strongly depends on the parameters of new physics, and can turn out to be even shorter than the age of the Universe.

Moreover, by comparing (4.4) and (4.5) we see that gravity, while still showing a slight tendency toward stabilization (which is what we observe in the absence of NP when comparing (2.27) with (2.31)), only produces a "tiny" effect, that qualitatively does not modify significantly the stability condition of the EW vacuum.

Despite the fact that in our toy model NP lives at very high energy scales, the expectation that the tunneling time should be insensitive to it, in other words that the result shown in (2.31) should not be modified by the presence of NP at high energies, is not fulfilled. These results confirm the analysis of the previous section. Here, with the help of a fully renormalizable toy UV completion of the SM, we have shown that the EW vacuum lifetime strongly depends on NP even if the latter lives at very high energy scales.

These findings are at odds with a widely diffused expectation, based on a naive application of the decoupling argument, and show that the fact that the vacuum stability condition depends on physics that lives at very high energy scales is not due to an illegitimate extrapolation of the theory beyond its validity, as it was previously thought [72]. On the contrary, that expectation was based on an illegitimate application of the decoupling theorem to a phenomenon (the tunneling of the EW vacuum) to which it cannot be applied.

Before ending this section, we would like to stress once again that with respect to the previous section, where NP interactions were parametrized with the help of higher order non-renormalizable operators, here NP is given in terms of a fully renormalizable theory, thus showing that the effect that we present is a genuine physical effect and has nothing to do with the specific parametrization of NP.

\section{Summary, conclusions, and outlook}

We studied the impact of very high energy NP (around the Planck scale $M_{\mathrm{P}}$ ) on the stability condition of the EW vacuum by carrying the analysis in a curved spacetime background, i.e. by taking into account the presence of gravity. We found that having included gravity in the stability analysis does not act against the formation of new vacuum bubbles, thus confirming the results of previous studies [38-40,43], where the analysis was carried in a flat spacetime background. As for this latter case, these new bounce solutions can have an enormous impact on the EW vacuum lifetime, by far dominating over the contribution that comes from the known solutions obtained with the unmodified Standard Model potential.

As in [38-40] we first performed the analysis by adding to the SM potential higher powers of the Higgs field, more precisely terms as $\phi^{6} / M_{\mathrm{P}}^{2}$ and $\phi^{8} / M_{\mathrm{P}}^{4}$ (section 3 ) that are certainly generated in a quantum gravity context [73]. Following [43] we then parametrized high energy new physics in a different manner, namely by adding to the SM potential a boson $S$ and a fermion $\psi$, with very large masses $M_{S}$ and $M_{f}$, coupled to the Higgs boson. 
As for the analysis carried in flat spacetime, in both cases we find that the presence of new physics can have an enormous impact on the EW vacuum stability condition.

These results definitely show that, irrespectively of the parametrization used to describe high energy new physics, it is not possible to ignore its presence when the stability analysis is performed. They are of the greatest importance for current studies and for model building of Beyond Standard Model physics, where we often have to take into account new physics at very high (Planckian and/or trans-Planckian) scales.

A question that is left open by the present analysis is the role that could be played by a non-minimal coupling of gravity to the Higgs boson. Work in this direction is in progress [74].

\section{Acknowledgments}

This work is carried out within the INFN project QFT-HEP and is supported in part by the HARMONIA project under contract UMO-2015/18/M/ST2/00518 (2016-2019). EB is also supported by the project "Digitizing the universe: precision modelling for precision cosmology", funded by the Italian Ministry of Education, University and Research (MIUR).

\section{A Numerical computation of the bounce solution}

The search for the bounce solution is a boundary-value problem specified by the values of $\varphi^{\prime}(0)$ and $\varphi(\infty)$. This can be turned into an initial-value problem using the shooting method, whereby $\varphi(\infty)$ is replaced by $\varphi(0)$, and the appropriate value for the latter quantity is found iteratively, solving eq. (2.23) (or its curved-space generalization) for different initial values until the desired $\varphi(\infty)$ is obtained.

As will be clear below, knowledge of the asymptotic behavior of $\varphi(x)$ for $x \rightarrow 0$ and $x \rightarrow$ $\infty$ is a crucial ingredient for the efficiency of the shooting algorithm. To find the expected behavior of $\varphi(x)$ in the relevant regimes, we begin by expanding $\varphi(x)$ around $x=0$ :

$$
\varphi(x)=B_{0}+B_{2} x^{2}+B_{3} x^{3}+\ldots
$$

where the linear term is missing due to the condition $\varphi^{\prime}(0)=0$. Inserting this expansion in (2.23), with $U(\varphi)$ given by (2.25) we find that the coefficients of the odd-power terms vanish, $B_{2 n+1}=0$, while all the coefficients of the even-power terms $B_{2 n}$ are functions of the first coefficient $B_{0}$ (called $B$ from now on). Truncating the expansion to the $x^{2}$ term:

$$
\varphi(x)=B+\frac{B^{3}}{8}\left(\lambda_{*}+\frac{\alpha}{2} \ln B+\alpha \ln ^{2} B+\beta \ln ^{3} B+\beta \ln ^{4} B\right) x^{2}+\ldots
$$

The coefficient of $x^{2}$ turns out to be negative, so near the origin the bounce profile behaves as an upside-down parabola.

As for the behavior of $\varphi(x)$ for $x \rightarrow \infty$, we note that $U(\varphi) \rightarrow 0$ for $x \rightarrow \infty$, and $\varphi(x) \rightarrow 0$ for $x \rightarrow \infty$. Asymptotically eq. (2.23) and the corresponding solution are then:

$$
\varphi^{\prime \prime}(x)+\frac{3}{x} \varphi^{\prime}(x)=0 \Rightarrow \varphi(x)=\frac{A}{x^{2}},
$$


where $A$ is one of the integration constants, while the second additive integration constant vanishes due to the condition $\varphi(\infty)=0$. In other words, for the bounce solution $x^{2} \varphi(x)$ has to reach a plateau for $x \rightarrow \infty$.

Numerically, we have implemented a fully adaptive algorithm designed to: (i) pick an initial guess $\left(\varphi(0)=B, \varphi^{\prime}(0)=0\right)$, (ii) integrate eq. (2.23) numerically while monitoring the behavior of $\varphi(x)$, and (iii) iteratively restart the procedure with a suitably corrected $B$ until the condition $\varphi(\infty)=0$ is satisfied up to a prescribed tolerance. In practice, the numerical integration is carried out in the range $\left[x_{\min }, x_{\max }\right]$, where we have chosen $x_{\min }=10^{-10}$ and $x_{\max }=10^{9}$, so that the initial conditions for $\varphi$ and $\varphi^{\prime}$ are given at $x=x_{\min }$ using eq. (A.2). With this boundary conditions, we find a class of solutions $\varphi_{B}(x)$, parametrized by $B$. Following the overshoot-undershoot argument of Coleman [53, 54], we want to tune the parameter $B$ until we converge to the solution which reaches a plateau for $x \rightarrow \infty$.

We found that the characterization of the final state is of crucial importance to the effectiveness of the search. In particular, introducing the reference point $x_{\text {ref }}=x_{\text {max }}-10^{3}$ and a tolerance $\epsilon=10^{-10}$, we found that the following three criteria are sufficient to lead the algorithm to the bounce solution in all cases (denoting $\tilde{\varphi}(x)=x^{2} \varphi(x)$ ):

1. If $\tilde{\varphi}\left(x_{\max }\right)-\tilde{\varphi}\left(x_{\mathrm{ref}}\right)>\epsilon$, the scalar field has reversed its direction before reaching $\varphi=0$, and is returning towards its initial position. The initial guess for $\varphi\left(x_{\min }\right)$ was therefore too low, and $B$ is correspondingly increased by a quantity $\delta$.

2. If $\tilde{\varphi}\left(x_{\max }\right)-\tilde{\varphi}\left(x_{\mathrm{ref}}\right)<-\epsilon$, the scalar field is overshooting the top of the hill. The initial guess for $\varphi\left(x_{\min }\right)$ was therefore too high, and $B$ is correspondingly decreased by a quantity $\delta$.

3. If $\left|\tilde{\varphi}\left(x_{\max }\right)-\tilde{\varphi}\left(x_{\mathrm{ref}}\right)\right|<\epsilon$, we consider that the plateau has been reached, i.e. that the bounce solution is found within the required precision.

Furthermore, the algorithm stores a state consisting of both $B$ and its value for the two preceding iterations. It is therefore possible to detect oscillations in $B$ and bisect (or otherwise decrease) $\delta$. We found that decreasing $\delta$ to $\delta / 10$ each time $B$ changes trend leads to a particularly efficient search, that converges exponentially to the solution, as will be illustrated below.

The inclusion of gravity does not modify our algorithm, as we found that, for $x \rightarrow \infty$, the areal radius goes as $a \sim x+c$ (see text), i.e. the curvature tends asymptotically to zero (the value of the constant $c$ is given by $a(x)-x$ when the plateau is reached).

For this reason, the criteria for tuning the initial value of $\varphi$, introduced in the flat case, can also be used on a curved spacetime. Again, we implemented the boundary condition in $x_{\text {min }}$ :

$$
\varphi\left(x_{\min }\right)=B \quad \varphi^{\prime}\left(x_{\min }\right)=0 \quad a\left(x_{\min }\right)=\epsilon^{\prime} \quad a^{\prime}\left(x_{\min }\right)=1,
$$

with $\epsilon^{\prime} \ll 1$ (clearly we cannot use $a\left(x_{\min }\right)=0$ due to the factor $a^{-1}$ in $(2.28 \mathrm{a})$ ).

After solving the equations, the size $\mathcal{R}$ of the bounce solution is obtained and we can compute the integral in eq. (2.30). 
We go now back to the flat spacetime case and include the NP terms of (3.1), thus obtaining the (dimensionless) potential (3.2). The bonus for our analysis is that this potential does not modify the asymptotic behavior of the bounce solution $\varphi_{\mathrm{b}}(x)$ for $x \rightarrow \infty$, as in this limit we still have $U(\varphi(x)) \rightarrow 0$. Therefore the inclusion of NP does not lead to any substantial change in our numerical method. The only modification with respect to the flat spacetime case concerns the expansion of the bounce solution around the origin $x=0$. In the flat case, by considering the integration range $\left[x_{\min }, x_{\max }\right]$, we found that the initial values for solving eq. (2.2) with the shooting method are obtained once we take the expansion (A.2) of $\varphi(x)$ and its first derivative at $x_{\min }$. Repeating the same analysis for the potential (3.2), we find that NP simply leads to additional terms in these expressions. Thus, the new expansion for $\varphi(x)$ is given by (again up to $O\left(x^{2}\right)$ ):

$$
\varphi(x)=B+\frac{B^{3}}{8}\left(\lambda_{*}+\lambda_{6} B^{2}+\lambda_{8} B^{4}+\frac{\alpha}{2} \ln B+\alpha \ln ^{2} B+\beta \ln ^{3} B+\beta \ln ^{4} B\right) x^{2}+\cdots
$$

which we use to set initial conditions for $\varphi\left(x_{\min }\right)$ and $\varphi^{\prime}\left(x_{\min }\right)$. An analogous approach is followed when we consider the alternative parametrization of NP given in eq. (4.3). Just like in the case without new physics, when we include gravity we use the initial values (A.4), and the numerical integration of the equations of motion is reduced to the tuning of the parameter $B$.

Figure 6 illustrates the exponential convergence of our algorithm in the four cases presented in this appendix.

Open Access. This article is distributed under the terms of the Creative Commons Attribution License (CC-BY 4.0), which permits any use, distribution and reproduction in any medium, provided the original author(s) and source are credited. 

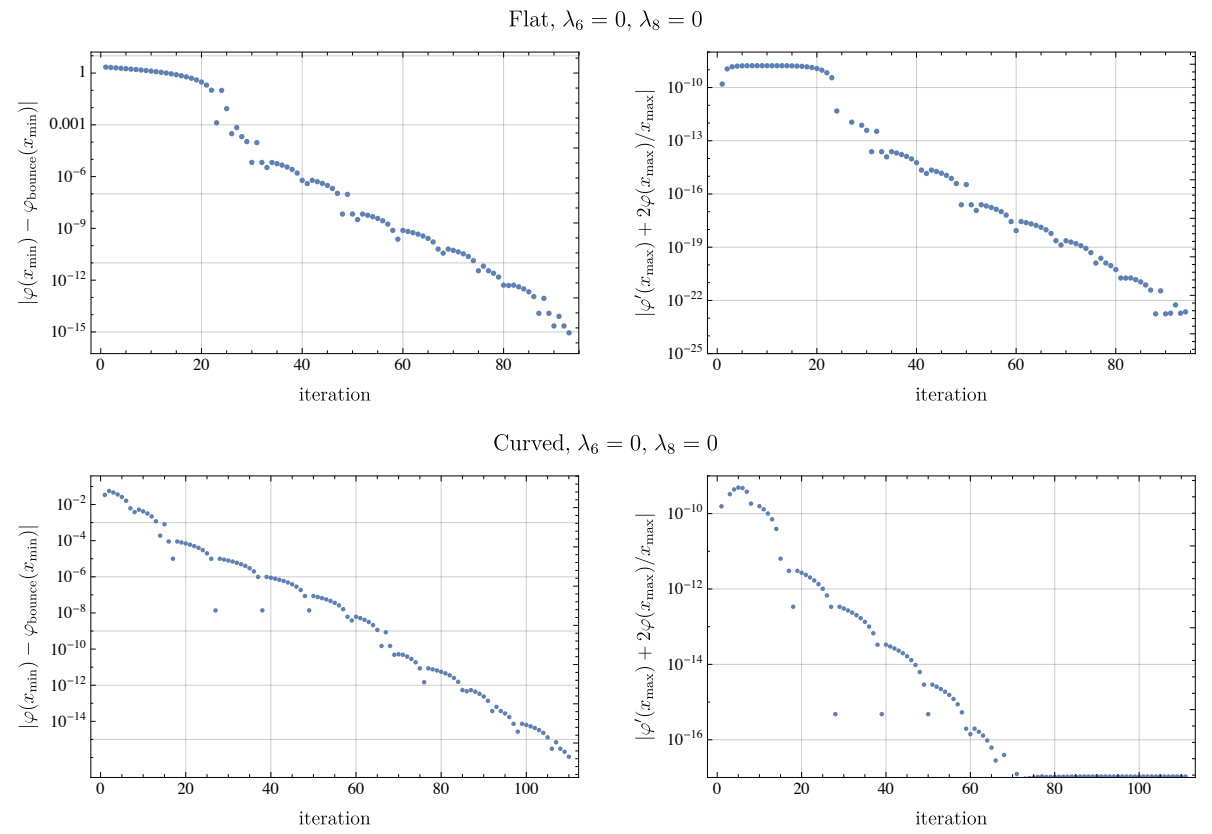

Flat, $\lambda_{6}=-1.2, \lambda_{8}=1$
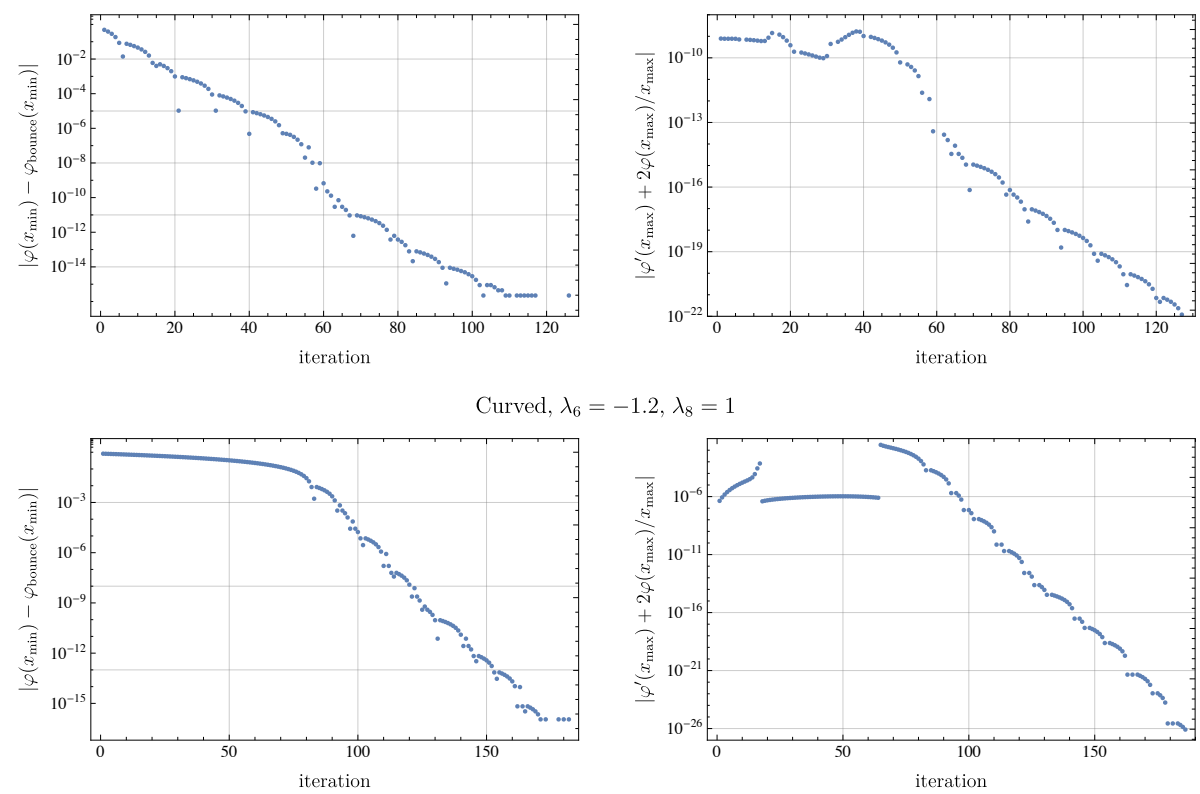

Figure 6. Convergence of $\varphi\left(x_{\min }\right)$ to its final value (left column), as well as of $\varphi^{\prime}\left(x_{\max }\right)+$ $2 \varphi\left(x_{\max }\right) / x_{\max }$ to zero (right column), in the four cases (flat and curved, with and without new physics) discussed in the appendix.

\section{References}

[1] N. Cabibbo, L. Maiani, G. Parisi and R. Petronzio, Bounds on the Fermions and Higgs Boson Masses in Grand Unified Theories, Nucl. Phys. B 158 (1979) 295 [InSPIRE].

[2] R.A. Flores and M. Sher, Upper Limits to Fermion Masses in the Glashow-Weinberg-Salam Model, Phys. Rev. D 27 (1983) 1679 [INSPIRE]. 
[3] M. Lindner, Implications of Triviality for the Standard Model, Z. Phys. C 31 (1986) 295 [INSPIRE].

[4] M. Sher, Electroweak Higgs Potentials and Vacuum Stability, Phys. Rept. 179 (1989) 273 [INSPIRE].

[5] M. Lindner, M. Sher and H.W. Zaglauer, Probing Vacuum Stability Bounds at the Fermilab Collider, Phys. Lett. B 228 (1989) 139 [INSPIRE].

[6] C. Ford, D.R.T. Jones, P.W. Stephenson and M.B. Einhorn, The Effective potential and the renormalization group, Nucl. Phys. B 395 (1993) 17 [hep-lat/9210033] [INSPIRE].

[7] M. Sher, Precise vacuum stability bound in the standard model, Phys. Lett. B 317 (1993) 159 [hep-ph/9307342] [INSPIRE].

[8] G. Altarelli and G. Isidori, Lower limit on the Higgs mass in the standard model: An Update, Phys. Lett. B 337 (1994) 141 [INSPIRE].

[9] J.A. Casas, J.R. Espinosa and M. Quirós, Improved Higgs mass stability bound in the standard model and implications for supersymmetry, Phys. Lett. B 342 (1995) 171 [hep-ph/9409458] [INSPIRE].

[10] J.A. Casas, J.R. Espinosa and M. Quirós, Standard model stability bounds for new physics within LHC reach, Phys. Lett. B 382 (1996) 374 [hep-ph/9603227] [INSPIRE].

[11] G. Isidori, G. Ridolfi and A. Strumia, On the metastability of the standard model vacuum, Nucl. Phys. B 609 (2001) 387 [hep-ph/0104016] [INSPIRE].

[12] J.R. Espinosa, G.F. Giudice and A. Riotto, Cosmological implications of the Higgs mass measurement, JCAP 05 (2008) 002 [arXiv:0710.2484] [INSPIRE].

[13] B.-H. Lee and W. Lee, Vacuum bubbles in a de Sitter background and black hole pair creation, Class. Quant. Grav. 26 (2009) 225002 [arXiv:0809.4907] [INSPIRE].

[14] B.-H. Lee, W. Lee, C. Oh, D. Ro and D.-h. Yeom, Fubini instantons in curved space, JHEP 06 (2013) 003 [arXiv: 1204.1521] [INSPIRE].

[15] B.-H. Lee, W. Lee, D. Ro and D.-h. Yeom, Oscillating Fubini instantons in curved space, Phys. Rev. D 91 (2015) 124044 [arXiv:1409.3935] [INSPIRE].

[16] M.S. Turner and F. Wilczek, Is our vacuum metastable?, Nature 298 (1982) 633 [INSPIRE].

[17] P. Hut and M.J. Rees, How stable is our vacuum?, Nature 302 (1983) 508 [INSPIRE].

[18] G. Degrassi et al., Higgs mass and vacuum stability in the Standard Model at NNLO, JHEP 08 (2012) 098 [arXiv: 1205.6497] [INSPIRE].

[19] B. Garbrecht and P. Millington, Green's function method for handling radiative effects on false vacuum decay, Phys. Rev. D 91 (2015) 105021 [arXiv:1501.07466] [INSPIRE].

[20] B. Grinstein and C.W. Murphy, Semiclassical Approach to Heterogeneous Vacuum Decay, JHEP 12 (2015) 063 [arXiv: 1509.05405] [INSPIRE].

[21] B. Garbrecht and P. Millington, Self-consistent radiative corrections to false vacuum decay, J. Phys. Conf. Ser. 873 (2017) 012041 [arXiv:1703.05417] [INSPIRE].

[22] M. Herranen, T. Markkanen, S. Nurmi and A. Rajantie, Spacetime curvature and the Higgs stability during inflation, Phys. Rev. Lett. 113 (2014) 211102 [arXiv:1407.3141] [INSPIRE].

[23] N. Khan and S. Rakshit, Study of electroweak vacuum metastability with a singlet scalar dark matter, Phys. Rev. D 90 (2014) 113008 [arXiv:1407.6015] [INSPIRE]. 
[24] M. Herranen, T. Markkanen, S. Nurmi and A. Rajantie, Spacetime curvature and Higgs stability after inflation, Phys. Rev. Lett. 115 (2015) 241301 [arXiv: 1506. 04065] [INSPIRE].

[25] J. Kearney, H. Yoo and K.M. Zurek, Is a Higgs Vacuum Instability Fatal for High-Scale Inflation?, Phys. Rev. D 91 (2015) 123537 [arXiv:1503.05193] [INSPIRE].

[26] L.A. Anchordoqui et al., Majorana dark matter through the Higgs portal under the vacuum stability lamppost, Phys. Rev. D 92 (2015) 063504 [arXiv:1506.04702] [InSPIRE].

[27] F. Kahlhoefer and J. McDonald, WIMP Dark Matter and Unitarity-Conserving Inflation via a Gauge Singlet Scalar, JCAP 11 (2015) 015 [arXiv: 1507.03600] [InSPIRE].

[28] Y. Ema, K. Mukaida and K. Nakayama, Fate of Electroweak Vacuum during Preheating, JCAP 10 (2016) 043 [arXiv: 1602.00483] [INSPIRE].

[29] Y. Ema, K. Mukaida and K. Nakayama, Electroweak Vacuum Stabilized by Moduli during/after Inflation, Phys. Lett. B 761 (2016) 419 [arXiv:1605.07342] [INSPIRE].

[30] N. Okada and D. Raut, Running non-minimal inflation with stabilized inflaton potential, Eur. Phys. J. C 77 (2017) 247 [arXiv: 1509.04439] [INSPIRE].

[31] K. Urbanowski, Properties of the false vacuum as a quantum unstable state, Theor. Math. Phys. 190 (2017) 458 [arXiv: 1609.03382] [INSPIRE].

[32] A. Stachowski, M. Szydłowski and K. Urbanowski, Cosmological implications of the transition from the false vacuum to the true vacuum state, Eur. Phys. J. C 77 (2017) 357 [arXiv: 1609.09828] [INSPIRE].

[33] D. Buttazzo et al., Investigating the near-criticality of the Higgs boson, JHEP 12 (2013) 089 [arXiv: 1307.3536] [INSPIRE].

[34] L.N. Mihaila, J. Salomon and M. Steinhauser, Gauge Coupling $\beta$-functions in the Standard Model to Three Loops, Phys. Rev. Lett. 108 (2012) 151602 [arXiv:1201.5868] [INSPIRE].

[35] K.G. Chetyrkin and M.F. Zoller, Three-loop $\beta$-functions for top-Yukawa and the Higgs self-interaction in the Standard Model, JHEP 06 (2012) 033 [arXiv: 1205.2892] [INSPIRE].

[36] F. Bezrukov, M.Y. Kalmykov, B.A. Kniehl and M. Shaposhnikov, Higgs Boson Mass and New Physics, JHEP 10 (2012) 140 [arXiv:1205.2893] [InSPIRE].

[37] J. Elias-Miro, J.R. Espinosa, G.F. Giudice, G. Isidori, A. Riotto and A. Strumia, Higgs mass implications on the stability of the electroweak vacuum, Phys. Lett. B 709 (2012) 222 [arXiv:1112.3022] [INSPIRE].

[38] V. Branchina and E. Messina, Stability, Higgs Boson Mass and New Physics, Phys. Rev. Lett. 111 (2013) 241801 [arXiv: 1307.5193] [INSPIRE].

[39] V. Branchina, E. Messina and A. Platania, Top mass determination, Higgs inflation and vacuum stability, JHEP 09 (2014) 182 [arXiv: 1407.4112] [INSPIRE].

[40] V. Branchina, E. Messina and M. Sher, Lifetime of the electroweak vacuum and sensitivity to Planck scale physics, Phys. Rev. D 91 (2015) 013003 [arXiv:1408.5302] [InSPIRE].

[41] N. Haba, H. Ishida, R. Takahashi and Y. Yamaguchi, Hierarchy problem, gauge coupling unification at the Planck scale and vacuum stability, Nucl. Phys. B 900 (2015) 244 [arXiv: 1412.8230] [INSPIRE].

[42] P.M. Ferreira and B. Swiezewska, One-loop contributions to neutral minima in the inert doublet model, JHEP 04 (2016) 099 [arXiv: 1511.02879] [INSPIRE]. 
[43] V. Branchina and E. Messina, Stability and UV completion of the Standard Model, Europhys. Lett. 117 (2017) 61002 [arXiv: 1507.08812] [INSPIRE].

[44] N. Chakrabarty and B. Mukhopadhyaya, High-scale validity of a two Higgs doublet scenario: metastability included, Eur. Phys. J. C 77 (2017) 153 [arXiv:1603.05883] [InSPIRE].

[45] A. Andreassen, W. Frost and M.D. Schwartz, Consistent Use of the Standard Model Effective Potential, Phys. Rev. Lett. 113 (2014) 241801 [arXiv: 1408.0292] [INSPIRE].

[46] L. Di Luzio and L. Mihaila, On the gauge dependence of the Standard Model vacuum instability scale, JHEP 06 (2014) 079 [arXiv:1404.7450] [INSPIRE].

[47] A. Andreassen, W. Frost and M.D. Schwartz, Consistent Use of Effective Potentials, Phys. Rev. D 91 (2015) 016009 [arXiv: 1408.0287] [INSPIRE].

[48] M. Endo, T. Moroi, M.M. Nojiri and Y. Shoji, False Vacuum Decay in Gauge Theory, JHEP 11 (2017) 074 [arXiv: 1704.03492] [INSPIRE].

[49] A. Andreassen, W. Frost and M.D. Schwartz, Scale Invariant Instantons and the Complete Lifetime of the Standard Model, arXiv:1707.08124 [INSPIRE].

[50] S. Chigusa, T. Moroi and Y. Shoji, State-of-the-Art Calculation of the Decay Rate of Electroweak Vacuum in Standard Model, Phys. Rev. Lett. 119 (2017) 211801 [arXiv: 1707.09301] [INSPIRE].

[51] T. Banks, C.M. Bender and T.T. Wu, Coupled anharmonic oscillators. I. Equal mass case, Phys. Rev. D 8 (1973) 3346 [InSPIRE].

[52] T. Banks and C.M. Bender, Coupled anharmonic oscillators. II. Unequal-mass case, Phys. Rev. D 8 (1973) 3366 [INSPIRE].

[53] S.R. Coleman, The Fate of the False Vacuum. I. Semiclassical Theory, Phys. Rev. D 15 (1977) 2929 [Erratum ibid. D 16 (1977) 1248] [INSPIRE].

[54] C.G. Callan Jr. and S.R. Coleman, The Fate of the False Vacuum. II. First Quantum Corrections, Phys. Rev. D 16 (1977) 1762 [inSPIRE].

[55] S.R. Coleman and F. De Luccia, Gravitational Effects on and of Vacuum Decay, Phys. Rev. D 21 (1980) 3305 [INSPIRE].

[56] ATLAS and CMS collaborations, Combined Measurement of the Higgs Boson Mass in pp Collisions at $\sqrt{s}=7$ and $8 \mathrm{TeV}$ with the ATLAS and CMS Experiments, Phys. Rev. Lett. 114 (2015) 191803 [arXiv:1503.07589] [INSPIRE].

[57] ATLAS, CDF, CMS and D0 collaborations, First combination of Tevatron and LHC measurements of the top-quark mass, arXiv:1403.4427 [INSPIRE].

[58] P.B. Arnold and S. Vokos, Instability of hot electroweak theory: bounds on $m_{H}$ and $m_{t}$, Phys. Rev. D 44 (1991) 3620 [INSPIRE].

[59] G. Isidori, V.S. Rychkov, A. Strumia and N. Tetradis, Gravitational corrections to standard model vacuum decay, Phys. Rev. D 77 (2008) 025034 [arXiv:0712.0242] [INSPIRE].

[60] V. Branchina, E. Messina and D. Zappalà, Impact of Gravity on Vacuum Stability, Europhys. Lett. 116 (2016) 21001 [arXiv: 1601.06963] [INSPIRE].

[61] A. Rajantie and S. Stopyra, Standard Model vacuum decay with gravity, Phys. Rev. D 95 (2017) 025008 [arXiv:1606.00849] [INSPIRE]. 
[62] A. Aguirre, T. Banks and M. Johnson, Regulating eternal inflation. II. The Great divide, JHEP 08 (2006) 065 [hep-th/0603107] [InSPIRE].

[63] R. Bousso, B. Freivogel and M. Lippert, Probabilities in the landscape: The Decay of nearly flat space, Phys. Rev. D 74 (2006) 046008 [hep-th/0603105] [INSPIRE].

[64] A. Masoumi, S. Paban and E.J. Weinberg, Tunneling from a Minkowski vacuum to an AdS vacuum: A new thin-wall regime, Phys. Rev. D 94 (2016) 025023 [arXiv:1603.07679] [INSPIRE].

[65] Y. Goto and K. Okuyama, Numerical analysis of Coleman-de Luccia tunneling, Int. J. Mod. Phys. A 31 (2016) 1650131 [arXiv:1601.07632] [INSPIRE].

[66] S.R. Coleman and E.J. Weinberg, Radiative Corrections as the Origin of Spontaneous Symmetry Breaking, Phys. Rev. D 7 (1973) 1888 [INSPIRE].

[67] O. Antipin, M. Gillioz, J. Krog, E. Mølgaard and F. Sannino, Standard Model Vacuum Stability and Weyl Consistency Conditions, JHEP 08 (2013) 034 [arXiv:1306.3234] [INSPIRE].

[68] P. Burda, R. Gregory and I. Moss, The fate of the Higgs vacuum, JHEP 06 (2016) 025 [arXiv: 1601.02152] [INSPIRE].

[69] J.R. Espinosa et al., The cosmological Higgstory of the vacuum instability, JHEP 09 (2015) 174 [arXiv: 1505. 04825] [INSPIRE].

[70] P.Q. Hung and M. Sher, Implications of a Higgs discovery at LEP, Phys. Lett. B 374 (1996) 138 [hep-ph/9512313] [INSPIRE].

[71] J.A. Casas, V. Di Clemente and M. Quirós, The Standard model instability and the scale of new physics, Nucl. Phys. B 581 (2000) 61 [hep-ph/0002205] [INSPIRE].

[72] J.R. Espinosa et al., The cosmological Higgstory of the vacuum instability, JHEP 09 (2015) 174 [arXiv: 1505. 04825] [INSPIRE].

[73] F. Loebbert and J. Plefka, Quantum Gravitational Contributions to the Standard Model Effective Potential and Vacuum Stability, Mod. Phys. Lett. A 30 (2015) 1550189 [arXiv: 1502.03093] [INSPIRE].

[74] E. Bentivegna, V. Branchina, F. Contino and D. Zappalà, work in progress. 